\title{
Étude comparée de l'hydrolyse du lactose \\ à l'aide des résines échangeuses d'ions \\ par voies enzymatique et catalytique
}

\author{
par \\ M. DEMAIMAY*, P. COMBEAUD*, A. GOUKASSOW* \\ et C. BARON** \\ (avec la collaboration technique de Nadine BEDUNEAU)
}

\section{INTRODUCTION}

Les procédés d'hydrolyse du lactose du lactosérum déprotéiné, qui conduisent à la production d'un mélange de sucres liquides, ouvrent une voie intéressante pour la revalorisation de ce produit en alimentation humaine.

Deux techniques semblent particulièrement bien adaptées à ce problème. La première est l'hydrolyse acide catalysée par une résine échangeuse de cations (R.E.C.) et la seconde l'hydrolyse enzymatique. Dans ce dernier cas, l'enzyme utilisée est la $\beta$-galactosidase qui est mise soit directement en solution avec le lactosérum, soit fixée sur un support poreux.

Différents supports de la $\beta$-galactosidase ont été testés : la cellulose [12]; le collagène [2]; les particules de verre poreux et de silice $[15,16]$; la chitine [14] ; le sphérosil [8] ; et les résines $[10,5,6,9]$.

Le complexe enzyme-support est avantageusement renforcé en utilisant le glutaraldéhyde comme agent de réticulation. L'étude du mécanisme d'immobilisation et son application à la trypsine ont été développées par Monsan [8].

La fixation de la B-galactosidase d'Aspergillus niger sur une résine du type phénol-formaldéhyde, réticulée avec du glutaraldéhyde,

* Laboratoire de Chimie-Biochimie Alimentaire (E.N.I.T.I.A.A.), La Géraudière 44072 Nantes cedex.

** Laboratoire de Biochimie (E.N.S.B.A.N.A.), Campus Universitaire - 21000 Dijon. 
pourrait être une des meilleures méthodes d'immobilisation testées, en particulier par Harju [6], qui utilise la résine Duolite S-761 et par Orok [11], avec la Duolite S-762.

Ici, en s'appuyant sur les données de l'hydrolyse catalytique, selon Demaimay [1], Haggett [4], et de l'hydrolyse enzymatique $[10,5,9,6,11]$, nous comparons d'une part l'efficacité des deux résines phénol-formaldéhyde S-761 et S-762, et développons le protocole opératoire qui semble le mieux adapté à l'hydrolyse du lactosérum déprotéiné à l'aide d'enzymes fixées sur ce type de support, d'autre part, cette méthode et les produits d'hydrolyse sont comparés au procédé et aux produits issus de l'hydrolyse acide catalysée par une résine échangeuse de cations (R.E.C.).

\section{MATERIEL ET METHODES}

\section{A. Matières premières}

- glutaraldéhyde, $\mathrm{C}_{5} \mathrm{H}_{8} \mathrm{O}_{2}$, en solution aqueuse à $25 \mathrm{p} .100$ (Prolabo) ;

- lactose monohydraté, $\mathrm{C}_{12} \mathrm{H}_{22} \mathrm{O}_{11}$, pour usages biochimiques (Merck) ;

- lactase d'Aspergillus niger, 200000 unités de O-nitrophényl- $\beta$-Dgalactopyranoside (O.N.P.G.) par gramme (Rapidase) ;

- lactosérum liquide déprotéiné et déminéralisé partiellement (Coopérative Laitière de la Prospérité Fermière, B.P. 34, 62001 Arras). Les caractéristiques du produit sont constantes et les suivantes : lactose $158 \mathrm{~g} / 1$, azote total 1,33 g/1, cendres $3,17 \pm 0,05 \mathrm{~g} / 1, \mathrm{pH} \mathrm{6,13}$ $\pm 0,02$;

- résines échangeuses d'ions (R.E.I.) de déminéralisation et d'hydrolyse catalytique : Société Applexion, Z.I. d'Epone - 78680 Epone ;

- R.E.I., support d'enzymes (Société Diaprosim, 107, rue EdithCaveil - 94400 Vitry-sur-Seine). Elles sont du type adsorbantes et en grains. La granulométrie de S-762 est plus fine que celle de S-761;

- sorbate de potassium (Labogros).

\section{B. Hydrolyse enzymatique}

$1^{\circ}$ Méthode De Fixation : fixation de $1 \mathrm{~g}$ de lactase sur $50 \mathrm{~g}$ de R.E.I.

a) A $1,5 \mathrm{~g}$ de lactase, sont ajoutés $10 \mathrm{ml}$ de tampon acétate $(0,01 \mathrm{M}, \mathrm{pH} 3,84)$. Cette solution est complétée à $150 \mathrm{ml}$ avec de l'eau distillée et ajustée à $\mathrm{pH} 4,1$ avec de l'acide chlorhydrique 0,04 N.

On laisse agir $100 \mathrm{ml}$ de la solution sur $50 \mathrm{~g}$ de résine pendant une nuit dans un bain-marie à $25^{\circ} \mathrm{C}$ sous agitation douce et constante. 
b) La solution d'enzyme surnageante est recueillie et son volume $\mathrm{V}_{0}$ est mesuré. La résine est lavée avec $200 \mathrm{ml}$ d'eau, cette opération est effectuée quatre fois et les volumes des eaux de rinçage $V_{1}, V_{2}$, $\mathrm{V}_{3}, \mathrm{~V}_{4}$ sont relevés.

Une solution ide glutaraldéhyde à 25 p. 100 dans l'eau distillée est ajoutée à la résine à raison de $1 \mathrm{ml}$ pour $10 \mathrm{ml}$ de résine. L'ensemble est maintenu $10 \mathrm{~h}$ à $0^{\circ} \mathrm{C}$ sous agitation.

Le surnageant de la solution de glutaraldéhyde est ensuite éliminé, puis on ajoute $400 \mathrm{ml}$ d'une solution de sorbate de potassium à 1 p. 100 (poids/volume).

On obtient ainsi un " complexe enzymatique » de type : résine$\beta$-galactosidase, réticulé par le glutaraldéhyde, qui se conserve à $4^{\circ} \mathrm{C}$.

\section{2o MESURE DE LA FIXATION}

Elle se fait au spectrocolorimètre à $400 \mathrm{~nm}$.

Le blanc est réalisé avec une solution de $10 \mathrm{ml}$ de tampon acétate, complété à $150 \mathrm{ml}$ avec de l'eau distillée.

Soit $\mathrm{D}_{\mathrm{i}}$, la densité optique de la solution d'enzyme à l'état initial, $\mathrm{C}_{\mathrm{i}}=10 \mathrm{~g} / \mathrm{l}$, la concentration.

Soit $\mathrm{D}_{0}$, la densité optique de la solution d'enzyme surnageante avant rinçage, et $D_{1}, D_{2}, D_{3}, D_{4}$, les densités optiques des différentes eaux de rinçage.

Remarque : il est indispensable de filtrer les échantillons sur papier filtre (Durieux no 111) avant chaque mesure pour éliminer le trouble.

\section{Calculs}

L'application de la loi de Beer-Lambert $(C=\varepsilon 1 C)$ nous permet d'établir une relation du type :

$$
C_{x}=C_{i} \cdot \frac{D_{x}}{D_{i}}
$$

avec $\mathrm{D}_{\mathrm{x}}: \mathrm{D}_{0}, \mathrm{D}_{1}, \mathrm{D}_{2}, \mathrm{D}_{3}, \mathrm{D}_{4}$.

La masse d'enzyme $\mathbf{M}_{x}$ dans chaque échantillon est alors :

$$
\mathrm{M}_{\mathrm{x}}=\mathrm{C}_{\mathrm{x}} \cdot \mathrm{V}_{\mathrm{x}}
$$

D'où : $\mathbf{M}=\mathbf{M}_{0}+\mathrm{M}_{1}+\mathrm{M}_{2}+\mathrm{M}_{3}+\mathrm{M}_{4}$.

Le poids initial étant de $1 \mathrm{~g}$, le pourcentage de fixation sera donc donné par la formule :

\section{$3^{\circ}$ Mesure de L'ACTIVITÉ}

$$
(1-M) \cdot 100
$$

Nous définirons ici l'activité comme le rapport du lactose hydrolysé par une certaine quantité d'enzyme fixée sur le lactose hydrolysé par la même quantité d'enzyme libre. 
Toutes les mesures sont effectuées à $37^{\circ} \mathrm{C}$ et sous agitation.

a) Enzyme libre

A $50 \mathrm{ml}$ d'une solution de $\beta$-galactosidase à 1,5 p. 100 dans un tampon acétate, on ajoute $200 \mathrm{ml}$ de lactose à $5 \mathrm{p} .100$.

On prélève $10 \mathrm{ml}$ aux temps $\mathrm{t}=2,5 \mathrm{~min}, 5 \mathrm{~min}, 7,5 \mathrm{~min}, 10 \mathrm{~min}$, $20 \mathrm{~min}, 30 \mathrm{~min}$ et on porte à $100^{\circ} \mathrm{C}$ pendant $3 \mathrm{~min}$, ce qui suffit à bloquer complètement l'action enzymatique.

Remarque: les essais effectués avec différents volumes de la solution d'enzyme libre montrent que c'est à partir de $50 \mathrm{ml}$ que l'on obtient un taux d'hydrolyse au moins égal à 60 p. 100 .

b) Enzyme fixée

Le calcul du pourcentage de fixation nous permet de prélever la quantité de résine humide qui a fixé $0,75 \mathrm{~g}$ de $\beta$-galactosidase.

A cette résine, on ajoute $200 \mathrm{ml}$ de lactose à 5 p. 100 et on opère comme précédemment.

40 INFLUENCE DE LA TEMPÉRATURE

a) Enzyme libre

Compte tenu du faible taux d'hydrolyse obtenu avec $50 \mathrm{ml}$ de solution d'enzyme libre, l'influence de la température est étudiée en ajoutant $100 \mathrm{ml}$ d'enzyme libre à $200 \mathrm{ml}$ d'une solution de lactose à 5 p. 100 .

b) Enzyme fixée

On dispose $700 \mathrm{~g}$ d'enzyme et son support dans une colonne de verre pyrex à double paroi qui est thermostatée à l'aide d'un cryostat (Haake FK2) contenant de l'éthylène glycol. Le débit de la solution est maintenu constant au moyen d'une pompe péristaltique à $0,26 \mathrm{~V} / \mathrm{V} / \mathrm{H}$ (volume par volume de " complexe enzymatique " et par heure).

Les taux d'hydrolyse du lactose sont déterminés par C.P.G. (voir paragraphe D).

5o PRotocole de L'HYdROLYSE ENZYMATIQUE DE LACTOSE DU LACTOSÉRUM

Le protocole de l'hydrolyse enzymatique met en œuvre :

- la colonne contenant le "complexe enzymatique ", en verre pyrex, à double paroi et thermostatée à $45^{\circ} \mathrm{C}$;

- le débit de la solution de lactosérum, fixé à $0,26 \mathrm{~V} / \mathrm{V} / \mathrm{H}$;

- la résine de déminéralisation cationique C.F. (Cation Fort) : fortement acide polystyrénique sulfoné ;

- et celle de déminéralisation anionique A.M. (Anion Moyen) : moyennement basique, polystyrénique. 


\section{Hydrolyse catalytique du lactose du lactosérum sur R.E.I.}

La solution de lactosérum est préchauffée à $95^{\circ} \mathrm{C}$ à l'aide d'un cryostat qui thermostatise aussi la colonne d'hydrolyse contenant une résine échangeuse d'ions du type cationique.

Il est toujours nécessaire de déminéraliser le lactosérum à l'aide de résines échangeuses d'ions.

On utilise à cet effet une résine cationique fortement acide (C.F.) et une résine anionique moyennement basique (A.M.).

\section{Technique d'analyse des sucres par C.P.G.}

Le chromatographe utilisé est un appareil Packard, modèle 419, équipé d'un détecteur à ionisation de flamme. L'intensité du détecteur est de 2,5.10-10 A. L'injection des échantillons se fait directement en tête de colonne à l'aide d'une seringue S.G.E de $5 \mu$ l. La colonne spiralée, en verre pyrex, a $3 \mathrm{~m}$ de long et $3 \mathrm{~mm}$ de diamètre interne, elle contient de la phase OV-17 à 5 p. 100 .

Support : Supelcoport 100-120 mesh.

La température de l'injecteur et du détecteur est de $250^{\circ} \mathrm{C}$.

Débits : gaz vecteur (azote : $30 \mathrm{ml} / \mathrm{min}$;

hydrogène : $15 \mathrm{ml} / \mathrm{min}$;

air : $300 \mathrm{ml} / \mathrm{min}$.

En nous appuyant sur la méthode de Zürcher [17], nous avons mis au point le protocole opératoire suivant : $5 \mu \mathrm{l}$ du produit à analyser sont mis à évaporer à sec à l'étuve à $80^{\circ} \mathrm{C}$. On y ajoute $50 \mu \mathrm{l}$ de pyridine et $100 \mu \mathrm{l}$ de N O-bis-(triméthylsilyl)-trifluoro-acétamide (B.S.T.F.A.) à 1 p. 100 de triméthylchlorosilane (T.M.C.S.).

Puis on porte à l'étuve $4 \mathrm{~h}$ à $80^{\circ} \mathrm{C}$.

Le dérivé silylé peut être conservé 3 semaines maximum à $4^{\circ} \mathrm{C}$.

Les conditions d'analyse sont les suivantes :

- température du four : $180^{\circ} \mathrm{C}$ pendant $10 \mathrm{~min}$ puis programmation de $5^{\circ} \mathrm{C} / \mathrm{min}$ jusqu'à $230^{\circ} \mathrm{C}$;

- injection de 1 à 2 ul de l'échantillon.

La durée d'une analyse est de $40 \mathrm{~min}$.

\section{E. Technique d'électrophorèse sur acétate de cellulose}

- appareil d'électrophorèse Desaga ;

- tampon véronal sodé à $8,24 \mathrm{~g} / 1 ; \mathrm{pH}=9,2$; force ionique $0,04 \mathrm{M}$;

- colorant : noir amido 1,25 g dans un mélange eau-acide acétique à 7 p. $100(500 \mathrm{ml})$;

- transparisant : méthanol/acide acétique/glycérol : 85/14/1 $(\mathrm{V} / \mathrm{V} / \mathrm{V})$. 
Sur les bandes d'acétate de cellulose imbibées de tampon, les échantillons sont déposés à l'aide d'un applicateur linéaire.

La tension est maintenue à $200 \mathrm{~V}$ pendant $1 \mathrm{~h}$. La fixation s'opère en immergeant dans un bain d'acide trichloracétique à 2,5 p. 100 pendant $5 \mathrm{~min}$.

Les bandes sont plongées dans le noir amido pendant $5 \mathrm{~min}$ et décolorées par trois ou quatre bains successifs d'acide acétique à 7 p. 100.

La transparisation est faite en déshydratant les bandes par immersion dans un bain de méthanol pur ( $3 \mathrm{~min}$ ). Plonger ensuite pendant $60 \mathrm{~s}$ dans la solution transparisante fraîchement préparée. Placer à l'étuve à $70^{\circ} \mathrm{C}$ jusqu'à transparence complète (3 à $5 \mathrm{~min}$ ).

\section{F. Dosage des matières azotées}

La minéralisation s'effectue selon la méthode de Kjeldahl.

L'ammoniac est déplacé du sel d'ammonium sous l'action de l'hydroxyde de sodium, distillé et recueilli dans une solution d'acide sulfurique.

L'excès d'acide sulfurique est titré par une solution d'hydroxyde de sodium en présence de rouge de méthyle.

\section{CHOIX DES MODALITES OPERATOIRES DE L'HYDROLYSE ENZYMATIQUE}

\section{A. Détermination des paramètres optimums de l'hydrolyse enzymatique}

Le produit final ne présente un intérêt technologique que si son taux d'hydrolyse est supérieur à 70 p. 100 .

\section{INFLUENCE DU TYPE DE RÉSINE}

Les résultats des études menées sur les résines S-761 et S-762 sont donnés dans le tableau 1. Ils mettent en évidence un meilleur pourcentage de fixation pour S-761. On note également que l'utilisation de quantités plus importantes de résine entraîne une chute sensible des pourcentages de fixation.

Quant à l'activité de la $\beta$-galactosidase, sur les deux types de résines, elle a été déterminée en établissant les courbes qui donnent le taux d'hydrolyse en fonction du temps, $H=f(T)$, et ceci en prenant comme référence l'enzyme libre en solution.

On observe que la résine S-762 est légèrement préférable avec un taux d'activité calculé à 30 min qui est de 83 p. 100 contre 80 p. 100 sur S-761. 
$T A B L E A U 1$

\begin{tabular}{l|rr|rr}
\hline $\begin{array}{c}\text { Quantité de } \\
\text { « complexe enzymatique " }\end{array}$ & \multicolumn{2}{|c|}{$50 \mathrm{~g}$} & & \multicolumn{2}{c}{$500 \mathrm{~g}$} \\
\cline { 2 - 5 } & & & & \\
Type de résine & S-761 & S-762 & S61 & S-762 \\
Pourcentage de fixation & 83,2 & 75,2 & 81,4 & 65,7 \\
\hline
\end{tabular}

Si l'on considère ces deux paramètres : fixation et activité, il apparaît une différence de réactivité entre les deux résines, S-761 fixe mieux l'enzyme, par contre le taux d'activité est légèrement supérieur avec S-762.

Si l'excès d'enzyme utilisé lors de la fixation peut être facilement récupéré et réutilisé, S-762 semble préférable compte tenu de son meilleur taux d'activité. Il est alors nécessaire de mettre en œuvre de plus grands volumes de résine afin de compenser son faible taux de fixation.

Si techniquement, ou économiquement, la réutilisation de l'excès d'enzyme est délicate, S-761 est alors mieux appropriée.

2० CHOIX DE LA TEMPÉRATURE

D'après K. Hyrkas [7], le pourcentage d'hydrolyse croît en fonction de la température jusqu'à $60^{\circ} \mathrm{C}$.

Mais, d'après Harju [5], la productivité du système d'hydrolyse décroît en fonction de l'élévation de la température par suite d'un début de dénaturation de l'enzyme.

Avec un débit fixé à $0,26 \mathrm{~V} / \mathrm{V} / \mathrm{H}$ et une solution en lactose de $\mathrm{pH} 4,1$, les essais montrent qu'une température minimum de $45^{\circ} \mathrm{C}$ permet d'obtenir un taux d'hydrolyse de 70,8 p. 100 , ce qui répond aux impératifs fixés. Une température supérieure entraîne des risques de dénaturation trop rapide.

\section{B. Protocole de l'hydrolyse enzymatique du lactose du lactosérum}

Les essais sont menés à $45^{\circ} \mathrm{C}$ avec un débit de $0,26 \mathrm{~V} / \mathrm{V} / \mathrm{H}$ et un lactosérum déprotéiné concentré à $158 \mathrm{~g} / 1$ de lactose. L'opération s'effectue avec $750 \mathrm{ml}$ de "complexe enzymatique ».

Avant passage, le $\mathrm{pH}$ du lactosérum est abaissé à 4,1 avec une solution d'acide acétique $(\mathrm{N})$. Cette acidification entraîne l'apparition d'un trouble que l'on attribue à la floculation des protéines du lactosérum qui n'ont pas été complètement éliminées lors des traite- 
ments d'ultrafiltration ou l'électrodialyse. Ce floculat a l'avantage d'être aisément filtrable.

Les dosages montrent qu'une faible proportion de matières minérales de l'ordre de 12,5 p. 100 est retenue sur le « complexe enzymatique ». On observe de plus une décoloration du produit en sortie de colonne d'hydrolyse qui est due à une adsorption irréversible des matières colorantes du lactosérum et en particulier de la riboflavine ou vitamine $\mathrm{B}_{2}$ sur le « complexe enzymatique ».

Or, l'adsorption des matières azotées, des sels minéraux et surtout des matières colorantes, entraîne rapidement une baisse d'efficacité du « complexe enzymatique » par colmatage des sites actifs.

C'est pourquoi nous proposons le procédé schématisé sur la figure 1, qui met en œuvre une résine cationique et une résine anionique de déminéralisation ; celle-ci présente les avantages suivants :

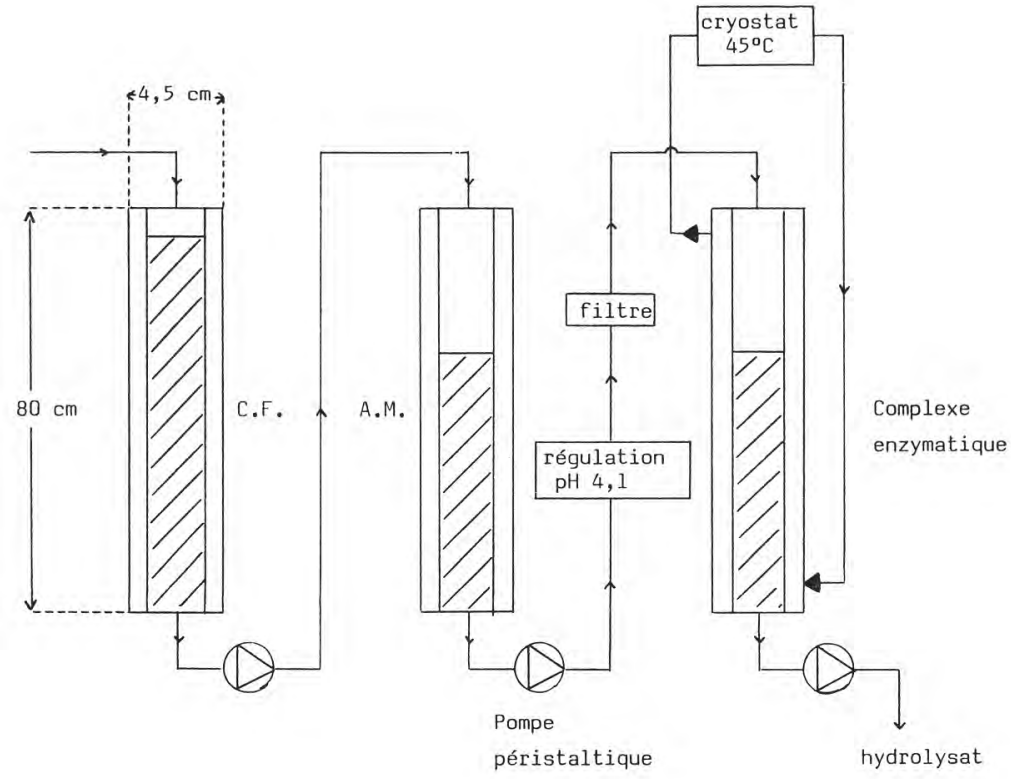

\begin{tabular}{|c|c|c|}
\hline $\begin{array}{c}\text { DEMINERAL ISATION } \\
\text { CATIONIQUE }\end{array}$ & $\begin{array}{c}\text { DEMINERALISATION } \\
\text { ANIONIQUE }\end{array}$ & $\begin{array}{c}\text { HYDROLYSE } \\
\text { ENZYMATIQUE }\end{array}$ \\
\hline
\end{tabular}

fig. 1

Procédé d'hydrolyse enzymatique du lactose

Hauteur des colonnes : $80 \mathrm{~cm}$.

Diamètre interne : $4,5 \mathrm{~cm}$. 
- premièrement, de déminéraliser efficacement le lactosérum puisque le pourcentage de cendres passe de 3,17 à 0,14 p. 100 ;

- deuxièmement, de fixer grâce aux propriétés adsorbantes des résines et plus particulièrement de la résine anionique les matières colorantes ;

- troisièmement, de piéger en moyenne 45 p. 100 des matières protidiques sur la colonne cationique et d'éviter en grande partie l'adsorption ultérieure de ces molécules sur le « complexe enzymatique ".

Ce procédé entraîne en outre la dilution du lactosérum donc un abaissement de la concentration en lactose, ce qui est favorable au taux d'hydrolyse puisque comme le montre Harju [5], ce taux diminue lorsque la concentration en lactose augmente. En l'occurrence, l'expérience montre ici que le taux d'hydrolyse obtenu est de 84 p. 100 alors que sans la dilution, il est de 74 p. 100.

\section{Etude des hydrolysats enzymatique et catalytique du lactosérum déprotéiné}

Les deux techniques qui sont testées avec le même lactosérum à $158 \mathrm{~g} / 1$ de lactose, un débit identique fixé à $0,26 \mathrm{~V} / \mathrm{V} / \mathrm{H}$, conduisent chacune à l'utilisation de trois colonnes, dont l'une est thermostatée à $95^{\circ} \mathrm{C}$ pour l'hydrolyse catalytique et à $45^{\circ} \mathrm{C}$ pour l'hydrolyse enzymatique.

Les deux produits obtenus sont incolores, limpides et de saveur sucrée, leurs caractéristiques sont données dans le tableau 2.

L'analyse chromatographique des hydrolysats permet de déterminer la quantité de lactose et de ses produits d'hydrolyse : glucose et galactose.

TABLEAU 2

\begin{tabular}{l|c|c}
\hline \multicolumn{1}{c|}{$\begin{array}{c}\text { Caractéristiques des } \\
\text { produits }\end{array}$} & $\begin{array}{c}\text { Hydrolysat catalytique } \\
\text { (H. C.) }\end{array}$ & $\begin{array}{c}\text { Hydrolysat enzymatique } \\
\text { (H.E.) }\end{array}$ \\
\hline pH & 6,7 & 4,7 \\
Matières sèches & $61 \mathrm{p} .100$ & $61 \mathrm{p} .100$ \\
Pourcentage taux d'hydrolyse & $95 \mathrm{p} .100$ & $84 \mathrm{p} .100$ \\
Azote total & $0,05 \mathrm{~g} / 1$ & $0,35 \mathrm{~g} / 1$ \\
\hline
\end{tabular}


Le taux d'hydrolyse reste plus élevé pour l'hydrolysat catalytique avec 95 p. 100 contre 84 p. 100 pour l'hydrolysat enzymatique.

Si l'on considère des hydrolysats enzymatique et catalytique à même taux d'hydrolyse, l'examen des chromatogrammes, figures 2 et 3, met en évidence la présence de di et tri-saccharides dans l'hydrolysat enzymatique qui n'apparaissent pas (ou seulement à l'état de traces) dans l'hydrolysat catalytique.

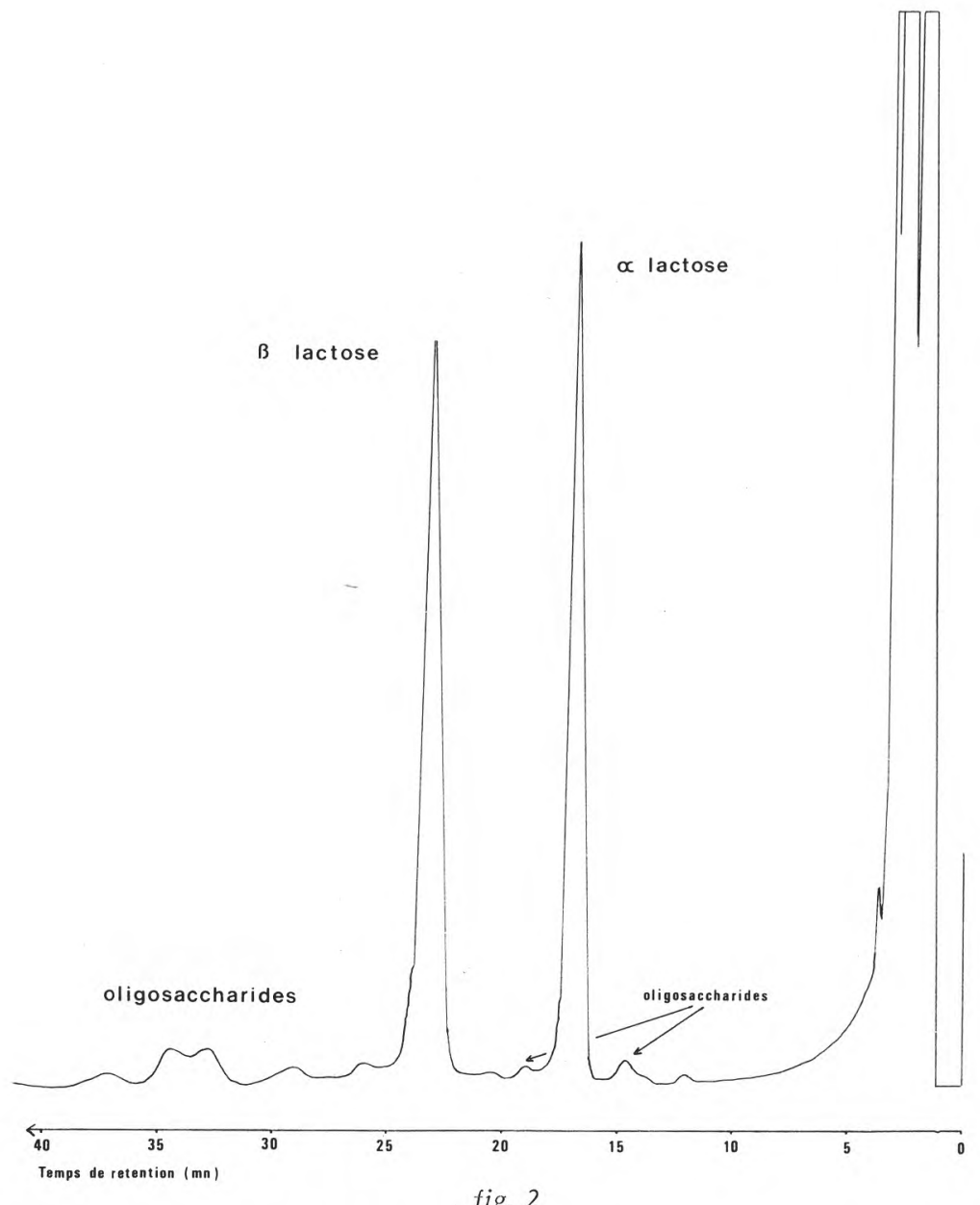

fig. 2

Chromatogramme d'un hydrolysat de lactosérum par voie enzymatique (enzymes fixées). Conditions d'analyse : cf. paragraphe II-D.

Les pics de glucose et de galactose sont ici confondus avec celui du solvant. 


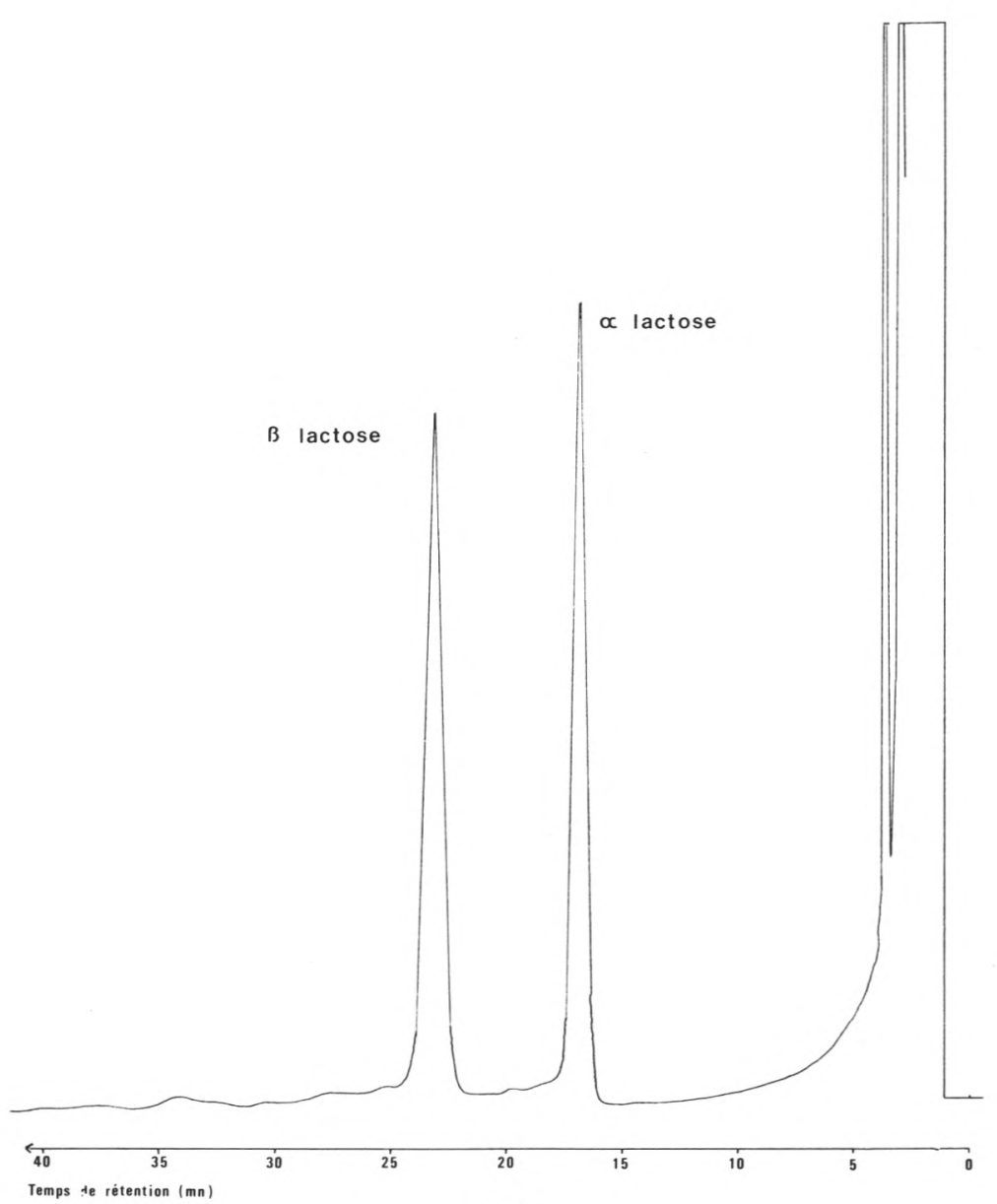

fig. 3

Chromatogramme d'un hydrolysat de lactosérum par voie catalytique. Conditions d'analyse : cf. paragraphe II-D.

Les pics de glucose et de galactose sont ici confondus avec celui du solvant.

On note toutefois que la quantité de polysaccharides obtenue par passage sur le "complexe enzymatique » est sensiblement plus faible que celle d'une hydrolyse enzymatique en réacteur [1].

Le temps de contact enzyme-lactose, qui est nettement plus long dans ce dernier cas, peut expliquer cette différence. 
TABLEAU 3

Hydrolyse catalytique (moyenne sur trois mesures)

\begin{tabular}{l|c|c}
\hline \multicolumn{1}{c|}{ Produits } & Azote total $\mathrm{g} / \mathrm{l}$ & $\begin{array}{c}\text { Pourcentage d'azote } \\
\text { fixé sur la colonne }\end{array}$ \\
\hline $\begin{array}{l}\text { Lactosérum de départ } \\
\begin{array}{l}\text { Sortie déminéralisation } \\
\text { cationique }\end{array}\end{array}$ & 1,33 & \\
$\begin{array}{l}\text { Sortie hydrolyse } \\
\begin{array}{l}\text { Sortie déminéralisation } \\
\text { anionique }\end{array}\end{array}$ & 0,74 & $45 \mathrm{p} .100$ \\
& 0,14 & $45 \mathrm{p} .100$ \\
\hline
\end{tabular}

Hydrolyse enzymatique (moyenne sur trois mesures)

\begin{tabular}{l|c|c}
\hline \multicolumn{1}{c|}{ Produits } & Azote total g/1 & $\begin{array}{c}\text { Pourcentage d'azote } \\
\text { fixé sur la colonne }\end{array}$ \\
\hline $\begin{array}{l}\text { Lactosérum initial } \\
\begin{array}{l}\text { Sortie déminéralisation } \\
\text { cationique }\end{array}\end{array}$ & 1,33 & \\
$\begin{array}{l}\text { Sortie déminéralisation } \\
\text { anionique }\end{array}$ & 0,75 & $45 \mathrm{p} .100$ \\
Sortie hydrolyse & 0,71 & $3,5 \mathrm{p} .100$ \\
& 0,35 & $27 \mathrm{p} .100$ \\
\hline
\end{tabular}

Les résultats des dosages d'azote total effectués au cours des différentes opérations d'hydrolyse sont donnés dans le tableau 3. Le lactosérum initial contient $1,33 \mathrm{~g} / 1$ d'azote total, qui se trouve essentiellement sous forme protidique.

Les deux procédés testés utilisent une colonne de déminéralisation cationique qui retient environ 45 p. 100 de cet azote. Il s'agit surtout de protides chargés positivement qui se fixent par échange d'ions sur la résine.

Quant à la fraction non fixée, les électrophorégrammes, figure 4, mettent en évidence que les protéines qui leur correspondent subis- 


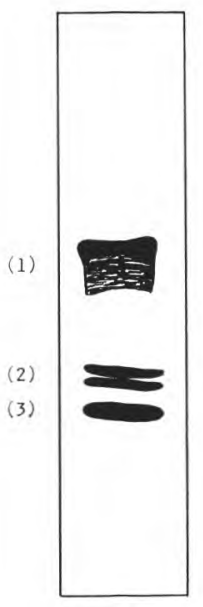

a

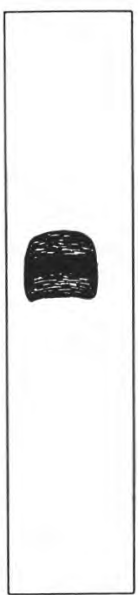

b

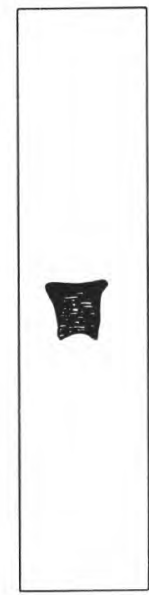

C

fig. 4

Electrophorèse sur bande de cellulose :

a) Mélange témoin :

$\beta$-lactoglobuline bovine (1) ;

$\gamma$-lactoglobulines dédoublées bovines (2);

sérumalbumine bovine (3).

b) Lactosérum avant passage sur la résine cationique de déminéralisation.

c) Lactosérum après passage sur la résine cationique de déminéralisation.

sent une modification de structure lors de leur passage sur la résine cationique. La forte acidité du milieu en est sans doute la cause et une hydrolyse partielle peut être envisagée.

Les deux procédés mettent également en œuvre une résine anionique qui fixe environ 3,5 p. 100 de matière azotée.

Avec le procédé catalytique, la résine d'hydrolyse piège ensuite une grande partie des protéines restantes, soit 45 p. 100 de l'azote total. Compte tenu de la nature cationique de la résine utilisée, il apparaît que c'est encore un mécanisme d'échange d'ions qui intervient ici. En conséquence, il est nécessaire, afin de maintenir l'activité catalytique de la résine, de la régénérer périodiquement à l'acide (tous les $40 \mathrm{~V} / \mathrm{V}$ environ).

L'analyse des 7,5 p. 100 d'azote qui restent dans l'hydrolysat catalytique indique qu'ils sont presque exclusivement sous forme de protéines et de peptides. En effet, sur $0,050 \mathrm{~g} / 1$ d'azote total, on trouve $0,003 \mathrm{~g} / 1 \mathrm{~d}$ 'azote aminé dosé par la méthode de Sörensen. 
Avec le procédé enzymatique, le pourcentage d'azote total retenu sur le "complexe enzymatique » est de 27 p. 100, donc sensiblement inférieur à celui obtenu par la méthode catalytique.

Le mécanisme de fixation repose sur une adsorption des protéines au niveau des grains de résine. La grande porosité de ces grains explique bien le phénomène.

Une élimination partielle des protéines peut être obtenue par un lavage à contre-courant du " complexe enzymatique ».

L'étude de la conservation des hydrolysats enzymatique et catalytique et en particulier de leur solubilité montre qu'un produit correspondant à un taux d'hydrolyse voisin de 80 p. 100 , avec un pourcentage en matières sèches entre 59 et 62 p. 100 , se conserve aisément pendant au moins 1 mois.

Ces données rejoignent les observations de Guy [3] et Shah [13]. En effet, en dessous de 75 p. 100 d'hydrolyse, la cristallisation du lactose est importante et au-dessus, la cristallisation du galactose est prédominante. Quant au taux de matières sèches, une concentration supérieure à 62 p. 100 entraîne en quelques jours la cristallisation des sucres des hydrolysats. Une concentration inférieure à 59 p. 100 amène des risques de pollution par micro-organismes.

\section{Rés u mé}

Si la $\beta$-galactosidase d'Aspergillus niger est mieux fixée sur la résine Duolite S-761, par contre, un taux d'activité légèrement meilleur est obtenu avec la Duolite S-762.

La mise en place du procédé d'hydrolyse enzymatique du lactose du lactosérum déprotéiné sur un complexe enzyme-résine réticulée avec le glutaraldéhyde, conduit à un système de déminéralisation cationique et anionique suivi de l'hydrolyse.

Outre la déminéralisation, la résine anionique assure la décoloration du produit en adsorbant la riboflavine et la résine cationique, qui fixe 45 p. 100 des matières protidiques, élimine leurs interférences au niveau du " complexe enzymatique ».

Les protéines non fixées subissent une modification de structure lors de leur passage sur la résine cationique qui peut être attribuée à une hydrolyse partielle.

La comparaison des produits d'hydrolyse enzymatique et catalytique montre que le complexe enzymatique fixe sensiblement moins de protides, avec 27 p. 100 contre 45 p. 100 avec la résine d'hydrolyse catalytique.

L'analyse en chromatographie gazeuse met en évidence des di et trisaccharides dans l'hydrolysat enzymatique, qui n'apparaissent que sous forme de faibles traces dans l'hydrolysat catalytique. 
Les deux hydrolysats obtenus sont incolores, limpides et de saveur sucrée. Un taux moyen d'hydrolyse de 80 p. 100 et un pourcentage de matières sèches compris entre 59 et 62 p. 100 permettent une bonne conservation du produit pendant au moins 1 mois.

\section{S u m m a r y}

COMPARATIVE STUDY OF HYDROLYSIS OF LACTOSE USING ION EXCHANGE RESINS BY ENZYMATIC AND CATALYTIC PROCESSUS

$\beta$-galactosidase of Aspergillus niger is better fixed on resin Duolite S-761, but a rate of activity slightly better is obtained with Duolite S-762.

The set up of the process of enzymatic hydrolysis of the lactose of deproteinated whey on a complex enzyme-resin reticulated with glutaraldehyde leads to a cation and anion demineralization system followed by hydrolysis.

Besides demineralization, the anion resin ensures decouloring of the product by adsorption of riboflavin, and the cation resin which fixes 45 p. 100 of protidic matter, eliminates its interference as regards the enzymatic complex.

When passing on the cation resin, non fixed proteins undergo a structural modification which may result from a partial hydrolysis.

The comparison of the products of enzymatic and catalytic hydrolysis shows that the enzymatic complex fixes quite a few less protids with 27 p. 100 versus 45 p. 100 with the resin of catalytic hydrolysis.

Gas liquid chromatography reveals di and tri-saccharides in the enzymatic hydrolysate, but these only appear as slight traces in the catalytic hydrolysate.

Both hydrolysates are colourless, limpid and sweet tasting. An average rate of hydrolysis of 80 p. 100 and a percentage of dry matter between 59 and 62 p. 100 allow a good preservation of the product for a least one month.

Reçu pour publication en août 1980

\section{Bibliographie}

[1] Dematmay (M.) (1977). - Etude comparative des produits d'hydrolyse enzymatique et catalytique du lactosérum déprotéiné sur résines échangeuses d'ions. Le Lait, (567), 351-361.

[2] Giacin (G. R.), Jakubowski (J.), Leeder (J. G.), Gilbert (S. G.) and Kleyn (D. H.) (1974). - Characterization of lactase immobilized on collagen: conversion of whey lactose by soluble and immobilized lactase. J. Food Science, 39, 751-754. 
[3] Guy (E. J.) and Edmonson (L. F.) (1978). - Preparation and properties of sirups made by hydrolysis lactose. J. Dairy Science, 61, 542-549.

[4] Haggett (T. O. R.) (1976). - The acid catalysed hydrolysis of lactose using a cation exchange resin. N.Z.J. Dairy Sci. Technol., 11, 176-179.

[5] Harju (M.), Heikonen (M.), Kreula (M.), Viskari (R.) and Linko (M.) (1978). - Hydrolysis of whey with immobilized lactase. First European Congress in Biotechnology. Interlaken, Pub. 2, 131-132.

[6] Har.Ju (M.) and Kreula (M.) (1978). - Lactose hydrolysates. N.J.F. Symposium on carbohydrate sweeteners, Espoo, Finland.

[7] Hyrkas (K.), Viskari (R.), Linko (Y. Y.) and Linko (M.) (1976). - Hydrolysis of lactose in acid whey by immobilized $\beta$-galactosidase. Milchwissenschaft, 31 , (3), 129-134.

[8] Monsan (P.) (1978). - Immobilisation d'enzymes à l'aide du glutaraldéhyde. Thèse d'Etat de l'Université Paul-Sabatier de Toulouse.

[9] Ohmiya (K.), Tanimura (S.), Kobayashi (T.) and Shimizu (S.) (1978). Preparation and properties of proteases immobilized on anion exchange resin with glutaraldehyde. Biotech. Bioeng., $X X, 1-15$.

[10] Olson (N.F.) and Richardson (T.) (1974). - Immobilized enzymes in food processing and analysis. J. Food Science, 39, 653-659.

[11] ORoK (M. R.), GrulKe (E. A.) and Syverson (A.) (1978). - Hydrolysis of lactose in acid whey using $\beta$-galactosidase adsorbed to a phenol formaldehyde resin. J. Food Science, 43, 566-571.

[12] Pastore (M.) Morisi (F.) and Viglia (A.) (1972). - Reduction of lactose of milk by entrapped $\beta$-galactosidase. II. Conditions for an industrial continuous process. J, Dairy Science, 57, 3, 269-272.

[13] Shah (N. O.) and Nickerson (T. A.) (1978). - Functional properties of hydrolyzed lactose: solubility, viscosity and humectant properties. J. Food Science, 43, 1081-1084.

[14] Stanley (W. L.), Watters (G. G.), Kelly (S. H.) and Olson (A. C.) (1978). Glucoamylase immobilized on chitin with glutaraldehyde. Biotech. Bioeng., $X X, 135-140$.

[15] Weetall (H.), Havewala (N. B.), Pitcher (W. H.), Detar (C. C.) Vann (W. P.). and YaVerbaum (S.) (1974). - The preparation of immobilized lactase and its use in the enzymatic hydrolysis of acid whey. Biotech. Bioeng., XVI, 295-313.

[16] Wierzbicki (L. E.) and Edwards (V. H.) (1974). - Hydrolysis of lactose in acid whey using $\beta$-galactosidase immobilized on porous glass particles: preparation and characterization of a reusable catalyst for the production of low-lactose dairy products. Biotech. Boieng., XVI, 397-411.

[17] Zurcher (K.), Hadorn (H.) und Strack (Ch.) (1975). - Gaschromatographische Zuckerbestimmung. Mitt. Gebiete Lebens. Hyg., 66, 92-116. 